tinct classes. According to our bound there are at least two more such splittings obtainable in this way.

\title{
REFERENCES
}

1. D. H. Lehmer, The Tarry-Escott problem, Scripta Math. vol. 13 (1947) pp. 3741.

2. J. B. Roberts, A curious sequence of signs, Amer. Math. Monthly vol. 64 (1957) pp. 317-322.

3. - A new proof of a theorem of Lehmer, Canad. J. Math. vol. 10 (1958) pp. 191-194.

4. E. M. Wright, Equal sums of like powers, Edinburgh Math. Proc. vol. 8 (1949) pp. $138-142$.

5. —, Equal sums of like powers, Bull. Amer. Math. Soc. vol. 54 (1948) pp. $755-757$.

6. - Prouhet's 1851 solution of the Tarry-Escott problem of 1910, Amer. Math. Monthly vol. 66 (1959) pp. 199-201.

Reed College

\section{A DETERMINANT CONNECTED WITH FERMAT'S LAST THEOREM}

L. CARLITZ ${ }^{1}$

Put

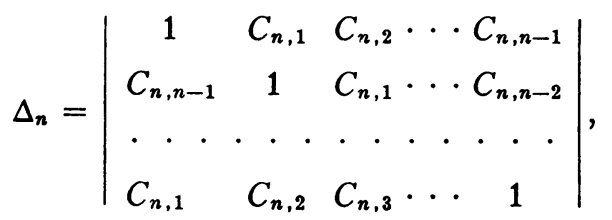

where the $C_{n, r}$ are binomial coefficients. Bachmann showed that if

$$
x^{p}+y^{p}+z^{p}=0
$$

is solvable then $\Delta_{p-1} \equiv 0\left(\bmod p^{3}\right)$. However Lubelski showed that for $p \geqq 7, \Delta_{p-1}$ is divisible by $p^{8}$, while E. Lehmer proved that $\Delta_{p-1}$ is divisible by $p^{p-2} q_{2}$, where $q_{2}=\left(2^{p-1}-1\right) / p$; also $\Delta_{n}=0$ if and only if $n=6 k$. For references see [2].

The writer [1] has determined the residue of $\Delta_{p-1}\left(\bmod p^{p-1}\right)$. The result is that

Received by the editors December 1, 1959.

1 Research supported by National Science Foundation, Grant G-9425. 


$$
\Delta_{p-1} \equiv p^{p-2} \prod_{a=1}^{p-2}\{(1+a) q(1+a)-a q(a)\}\left(\bmod p^{p-1}\right)
$$

where

$$
q(a)=\frac{a^{p-1}-1}{p}
$$

or if we prefer,

$$
\Delta_{p-1} \equiv \prod_{a=1}^{p-2}\left((a+1)^{p}-a^{p}-1\right)\left(\bmod p^{p-1}\right) .
$$

Now it is known (see [3, p. 564] for references) that when (1) is solvable

$$
q(r) \equiv 0(\bmod p)
$$

for all primes $r \leqq 43$ and therefore for all integral $r \leqq 46$. Mrs. Lehmer noted that it follows from

$$
q(2) \equiv 0(\bmod p)
$$

that if (1) is solvable then $\Delta_{p-1}$ is divisible by $p^{p-1}$. In view of (2) it seems plausible that when (1) is solvable $\Delta_{p-1}$ is divisible by a considerably higher power of $p$; however since the modulus in (2) is only $p^{p-1}$ such a result cannot be inferred without further proof.

Put $C_{r}=C_{p-1, r}$ for $0 \leqq r \leqq p-1$ and $C_{r}=C_{s}$ for $r \equiv s(\bmod p-1)$. Then

$$
\Delta_{p-1}=\left|C_{s-r}\right| \quad(r, s=1, \cdots, p-1) .
$$

Let $e$ be an arbitrary non-negative integer and consider the determinant

$$
D_{e}=\left|s^{p r r}\right| \quad(r, s=1, \cdots, p-1) .
$$

Then

$$
D_{e} \equiv D_{0}(\bmod p) ;
$$

since

$$
D_{0}=(p-1) ! \prod_{1 \leq r<s \leq p-1}(r-s),
$$

it follows that

$$
D_{e} \not \equiv 0(\bmod p) .
$$

Similarly the determinant 


$$
D_{c}^{\prime}=\left|r^{-p e s}\right| \quad(r, s=1, \cdots, p-1)
$$

is a rational number with both numerator and denominator prime to p. Consequently

$$
\Delta_{p-1}^{\prime}=D_{\bullet}^{\prime} \Delta_{p-1} D_{\bullet}
$$

and $\Delta_{p-1}$ are divisible by the same power of $p$.

We have

$$
D_{e}^{\prime} \Delta_{p-1} D_{e}=\left|A_{r s}\right| \quad(r, s=1, \cdots, p-1)
$$

where

$$
\begin{aligned}
& A_{r \varepsilon}=\sum_{j, k=1}^{p-1} r^{-p^{\star j}} C_{k-j} s^{p e s} \\
& =\sum_{t=1}^{p-1} C_{t} \sum_{k-j=t} r^{-p o i} s^{p e k} \\
& \equiv \sum_{t=1}^{p-1} C_{t} \sum_{j=1}^{p-1}\left(r^{-p 0} s^{p t}\right)^{i} s^{p t t}\left(\bmod p^{e+1}\right) .
\end{aligned}
$$

Since

$$
\sum_{j=1}^{p-1}\left(r^{-p o} s^{p e}\right)^{i} \equiv(p-1) \delta_{r \varepsilon}\left(\bmod p^{e+1}\right)
$$

where $\delta_{r s}$ is the Kronecker delta, we get

$$
\begin{aligned}
A_{r s} & \equiv(p-1) \delta_{r s} \sum_{t=1}^{p-1} C_{p-1, t} s^{p o t} \\
& \equiv(p-1) \delta_{r s}\left\{\left(1+s^{p 0}\right)^{p-1}-1\right\}\left(\bmod p^{e+1}\right) .
\end{aligned}
$$

Therefore (3) and (4) imply

$$
\Delta_{p-1}^{\prime} \equiv-(p-1)^{p-1} \prod_{r=1}^{p-2}\left\{\left(1+r^{p o}\right)^{p-1}-1\right\}\left(\bmod p^{e+1}\right) .
$$

Incidentally it is easily verified that

$$
D_{e}^{\prime} D_{c} \equiv(p-1)^{p-1}\left(\bmod p^{e+1}\right),
$$

so that

$$
\Delta_{p-1}^{\prime} \equiv(p-1)^{p-1} \Delta_{p-1}\left(\bmod p^{o+1}\right) .
$$

From (5) and (6) we get 


$$
\Delta_{p-1} \equiv-p^{p-2} \prod_{r=1}^{p-2} q\left(1+r^{p^{c}}\right)\left(\bmod p^{e+1}\right) .
$$

Now if (1) is solvable we have

$$
q(a) \equiv 0(\bmod p)
$$

Also if

$$
a^{p} \equiv a\left(\bmod p^{2}\right)
$$

it follows at once that

$$
\left(1+a^{p^{c}}\right)^{p-1} \equiv a^{p-1} \equiv 1\left(\bmod p^{2}\right) \quad(a<46),
$$

so that

$$
q\left(1+a^{p^{b}}\right) \equiv 0(\bmod p)
$$

for all $e \geqq 0$. Hence (since $p>50$ ) (7) yields

$$
\Delta_{p-1} \equiv c p^{p+43}\left(\bmod p^{e+1}\right),
$$

where $c$ is some integer. If we take

$$
e=p+42
$$

we obtain the following

THEOREM. If the equation

$$
x^{p}+y^{p}+z^{p}=0
$$

is solvable in rational integer $x, y, z$ each prime to $p$ then

$$
\Delta_{p-1} \equiv 0\left(\bmod p^{p+48}\right) .
$$

We remark that the theorem is meaningful only for $p \equiv-1(\bmod 6)$ since the determinant $\Delta_{p-1}$ is zero when $p \equiv 1(\bmod 6)$.

\section{REFERENCES}

1. L. Carlitz, A determinant connected with Fermat's last theorem, Proc. Amer. Math. Soc. vol. 10 (1959) pp. 686-690.

2. E. Lehmer, On a resultant connected with Fermat's last theorem, Bull. Amer. Math. Soc. vol. 41 (1935) pp. 864-867.

3. H. S. Vandiver, Fermat's last theorem. Its history and the nature of the known results concerning it, Amer. Math. Monthly vol. 43 (1946) pp. 555-578.

DURE UNIVERSITY 